\title{
Study on "VIS design" Textbook Construction in Higher Vocational Colleges in an Information Era
}

\author{
Feng Mao \\ Department of Applied Arts \\ Jianghai Polytechnic College \\ Yangzhou, Jiangsu, China 225002
}

\begin{abstract}
Arrival of the information age has changed the way of people to obtain visual information, and the previous traditional-media-dominated VIS design mode has not fully met the requirements for corporate image design in the information age, while VIS design gradually changed the single static transmission mode but brought a combined static and dynamic transmission mode. In response to this situation, the author sorted out the changes of VIS design content in the information age and put forward his concrete ideas of teaching textbook construction in step with the development of information age about how to stress Applied Teaching in the textbook construction with the "VIS design" course as the core curriculum of visual design specialty in higher vocational colleges.
\end{abstract}

Keywords-informatization; VIS design; textbook
construction

\section{INTRODUCTION}

VIS is the abbreviation of Visual Identity System. VIS design is to convey corporate spirit to the stakeholder of corporate and the society through the unified, systematic and normalized visual identity design, thus to establish the unified visual image [1]. VIS is of infection and transmissibility. A complete effective VIS design will render the corporate a decisive position in market economy, which is commonly called "face of corporate", becoming an important component in the development process of corporate and brand. As a result, necessary VIS design is an indispensable part of corporate long-term development. The main objective of higher vocational colleges is to cultivate application-oriented technical personnel. Therefore, the demands by market and the attention paid by corporate have make "VIS design course" the core course of visual design majors in higher vocational colleges. Since the introduction of modern VIS design into China in 1980s, it has been transmitted by print media in a static manner for quite a long time. So most of the textbook compilation for this course carries on the traditional VIS Manual's compiling system, starting form print media, and develop around basic system and application system. Along with the arrival of information era, new media technology has developed rapidly, and VIS system also changes along with the development of new media technology, turning to the transmission mode of combining dynamic and static from the pure static

Fund Project: The issues on education reform and textbook construction (No: 02-216) of Chinese Society of VTE in Year 2015-2016. transmission mode of print media in the past. The textbook construction for this course in higher vocational colleges is relatively lagged behind compared to VIS design content changes along with the development of information era. Most of the textbook contents stay in the VIS static framework, which cannot satisfy the demands by target position in information era against this course's textbook construction.

\section{IMPACT ON VIS DESIGN TEXTBOOK CONSTRUCTION OF HIGHER VOCATIONAL COLLEGES BY INFORMATION ERA}

Each great technology revolution will trigger an innovation within art design field. With the impact of new media technology, VIS design is undergoing an epochmaking change. The transmission speed of VIS design mode once based on such four traditional media as "television, broadcasting, newspapers and periodicals as well as outdoor media" is limited by the operating rules of traditional media itself, for example, the influences by print cycle, delivery and effectiveness and so on, which is of the limitation in terms of region and time. And its expression forms are quite simple, which is difficult to satisfy current society's demands for immediate, multi-dimensional and massive information transmission, and unable to completely meet the needs of corporate brand and image promotion under the background of information era.

In information era, new media technologies are developing rapidly, such as e-magazine, mobile terminal, LED...Internet and mobile communication. People's demand for information is also developing gradually following the changes in life style. It can be said that the biggest difference between new media and traditional media lies in the changes in contents, forms and transmission modes. The information transfer and communication on Internet just need to convert various information to digital signal, and it will be delivered to anywhere in the world. The change in presentation modes of new media may provide a wider and more tolerant presentation mode for VIS design. VIS design can present corporate image through real time application software APP, outdoor LED, QR Code Scan. Now, VIS design has entered into a new development stage along with the arrival of information era. Traditional VIS design has developed into a design system accustomed to new media transmission in information era. It is breaking 
traditional media scope and developing into multimedia. It exists in digital form and extends to multiple dimensions. The dynamic design of VIS system has become a trend, and various visual elements of VIS basic system, no matter the logo, standard character, standard color, mascot or the auxiliary graph, may generate dynamic changes according to the change of time and space; the stylized normative design of VIS application system is reducing gradually; the requirement of interactivity and adaptability is strengthened.

Most of the current textbooks as compiled for VIS Design course of higher vocational colleges tend to neglect the influence on VIS design contents by the appearance of new media, so the textbook contents are away from characteristics of information era. The textbook construction of VIS Design course for the higher vocational colleges with the aim of application oriented talents cultivation must insist on market-orientation, keep pace with the times, sort the changes as generated in VIS design basic system and application system in information era, as well as modify and rebuild the traditional textbooks on the basis of the aforementioned, make the textbook of VIS Design course of higher vocation colleges more complete and normal, and render it higher applicability and characteristics of times.

\section{CONSTRUCTION THOUGHTS OF VIS DESIGN TEXTBOOK's BASIC SYSTEM FOR HIGHER VOCATIONAL COLLEGES IN INFORMATION ERA}

Basic system is the basic element of VIS design, and the core part of VIS Design textbook contents. Its main content is how to design and regulate basic visual elements. Basic system includes the logo, standard character, standard color, mascot, auxiliary graph and so on. Among which, the logo, standard character and standard color are listed as the three cores of the system. Traditional VIS Design textbooks are compiled according to the aforementioned contents, which is the static standard against paper media primarily. Various visual element of VIS design in information era, no matter the logo, auxiliary elements or the color, may generate dynamic change according to changes in time and space. Therefore, the VIS Design textbook construction of higher vocational colleges in information era need more attention on the compatibility of basic elements, to make it not only be able to adapt to print media with various specifications, and electronic display mode with different resolution ratio, but also to formulate the usage regulations of its dynamic effects, reflecting the uniformity of VIS design system.

Logo is the symbolic identification symbol of a corporate and the core of VIS design. It is used most widely in VIS design basic system, and its frequency-of-occurrence is the highest. In traditional VIS Design textbook, as for logo design, it is often the static specification against print design demand. The modern multimedia devices emerge one after another, for example, such as the computer, WAP, multimedia projector and so on, have created conditions for logo's dynamic development. Dynamic logo is full of movement with the characteristics of variability. It is able to display the continuity and variability that cannot be expressed by the original static logo. As a result, this part of contents of VIS Design textbook for higher vocational colleges in informational era need to add design specification of dynamic design according to transmission mode and new media characteristics. For example, add the three most widely used logo specifications in Internet of $88 \times 31$ (pixel), $120 \times 60$ (pixel), $120 \times 90$ (pixel) to the textbook.

Standard character is the character specially used to reflect corporate name or brand, and it is used widely, which is one of the basic elements of VIS design. The compilation of contents about standard character in traditional VIS Design textbook is mainly starting from the perspective of print media, and regulates the standard character's stroke configuration and line weight according to the arrangement characteristics of four-color printing dot pattern. Under the background of information era, the problem that traditional printing specification used in digital media transmission may generate defects has appeared. Digital display conducts the display according to the screen, the size and density of each matrix's horizontal and vertical arrangement. For example, the elegant Song typeface in print media which is used widely will loss its beauty as its vertical line will be relatively thick and horizontal line will be unclear when the screen is reduced to a certain proportion. The annotation regulation for standard character in different media shall be added to the VIS Design textbook of higher vocational colleges in information era, and the importance of regulating harmony between new media display and print display shall be explained.

Standard color is specially designed according to the corporate image, and it is a kind or a group of specific colors which may represent the corporate concept, making corporate image maintain unity in terms of color. The compilation of contents about standard color in traditional VIS Design textbook is mainly starting from the perspective of print media, and regulates the color on the basis of CMYK (cyan, magenta, yellow and black). Under the background of information era, new carrier of electronic screen to display standard color has appeared, and it is defined based on the optical principles of color of RGB (red, green and blue). Electronic display browsing has the characteristics of jumping and transient of visual stay compared with print media. The principles for electronic screen and print media are different, as a result, the use of a set of standard color in electronic media and print media is totally different, as electronic screen require the standard color clearer and brighter. The contents of this part of VIS Design textbook for higher vocational colleges in information era shall take multiple requirements of the plane print and multimedia display into overall consideration against the changes in standard color's application carrier and the specificity of new media, and add the design specification of standard color in terms of page design or the page view on smart device, and make supplement of the knowledge about how to formulate a dual visual standard system suitable for both plane print and screen display with a set of standard color system.

The mascot of corporate shortens the distance between corporate and consumers with its affinity and interestingness, to intensify corporate character, and achieve a wider corporate image transmission effect. How to design and regulate the mascot's static modeling with different 
movements is the emphasis in compilation of traditional VIS Design textbook. In information era, the mascot image will not only appear on paper media in the manner of static form, but also appear on screen, network and mobile devices in dynamic form. The mascot may render a vivid display effect through dynamic demonstration. Therefore, not only the static content of mascot shall be compiled for the part of corporate mascot of VIS Design textbook for higher vocational colleges in information era, but the making specification of dynamic mascot design shall be added, to develop the mascot image full of dynamic demonstration effect [2], for example, to develop dynamic mascot with the form of interaction according to mobile media characteristics; develop the mascot animation with plot and social effect according to screen characteristics.

As part of VIS design, auxiliary graph may render richer and application of visual system with more levels. The compilation of auxiliary graph in traditional VIS Design textbook is made around the expression of auxiliary graph in print media. In information era, the contents regarding auxiliary graph in VIS Design textbook need a specification system for the use of auxiliary graph in new media [3], for example, the dynamic auxiliary graph applied to television advertising background, the static or dynamic auxiliary graph applied to corporate webpage background, as well as the auxiliary graph used in show background of multimedia courseware.

\section{CONSTRUCTION THOUGHTS OF VIS DESIGN TEXTBOOK'S APPLICATION SYSTEM FOR HIGHER VOCATIONAL COLLEGES IN INFORMATION ERA}

VIS application system is the specific specification made for the application of basic system in various media, which runs through every aspect of corporate work. Application system mainly includes office supplies system, environmental guide system, clothing and accessories system, propaganda system, public relation gifts system, and transportation system and so on. Traditional VIS application system is mainly the relevant hardware design for the corporate. However, under the background of information era, VIS application system pays more attention to the construction of soft facilities [4]. Therefore, corresponding adjustment shall be made for the compilation of the part of application system of the VIS Design textbook for higher vocational colleges.

Office supplies system is an important media for the corporate relationship with the outside. For example, business card, letter paper and various correspondence bills, although they are very small details, we can see big aspect of things from the small details. In the business association and public affairs between corporate and customers, as well as corporate and affiliated corporate, they may reflect the corporate unique and uniform image. The contents of office supplies in traditional VIS Design textbook generally include the design of business card, business card box, business card holder, invitation card, folder, contract and certificate, envelope, employee's card, letter of recommendation, note, and Fax paper and so on. The compilation basic is to conduct design and specification of paper, printing requirements (solid color, two-color, and four-color) and size with the principle of face-to-face interaction. In information era, along with the popularization of web conference and ecommerce, although the paper stationery with respect to office supplies not disappears completely, the frequency of using it has decreased significantly. Such supplies as E-mail, WeChat, and two dimensional codes have taken a more important role. Take business card as an example, the application of electronic business card is increasingly wide, and develops to dynamic using new media technology. As a result, as for compilation of office supplies of VIS Design Textbook for higher vocational colleges, the standard design of knowledge about E-Design shall be strengthened. For instance, add the image style design of homepage, corporate image style and format design within website pages (layout specification of website logo, standard character, standard color in website pages), electronic business card format design, E - MAIL digital letter format and use specification, corporate PPT presentation format, OFFICE document format, corporate computer boot screen, electronic desktop and so on to the textbook.

Environmental guide system includes internal environment and external environmental, with the purpose of keeping the internal and external environment of the corporate in order. Internal environment design of environmental guide system is guideline for the location of various departments and various facilities the corporate. And external environment design is the visual image representation of corporate image in public. The knowledge about environmental guide system in traditional VIS Design textbook generally includes such design of various doorplates, guiding sign, reception room background plate, and conference room background plate, as well as lounge and store image. Along with the appearance of new material and new production process, gone are the days when the traditional material and traditional process of Acrylic, bicolor board, paint and silkscreen are prevailing in the world. LCD screen and projection equipment have been widely used in environmental guide system. Therefore, the part of environmental guide of VIS Design textbook for higher vocational colleges shall be compiled with emphasis.

Clothing and accessories may improve employee's mental outlook, reflecting the overall strength of a corporate. The knowledge about clothing and accessories in traditional VIS Design textbook generally includes the design of ordinary staff uniform, administrative staff uniform, T-shirt, tie and safety helmet. In information era, the contents about clothing and accessories in VIS Design textbook for higher vocational colleges shall renew the manufacturing technology of clothing and pay attention to the changes in clothing style.

Propaganda system is a major means for information transmission of modern corporate, and the most direct and the most important way to promote corporate visual image. The knowledge of propaganda system in traditional VIS Design textbook generally includes the design of company guidelines, enterprise introduction, commodity instructions, internal publications, publicity magazines, Recruitment ads, newspaper ads, magazine ads, posters and TV advertising. 
The design of a lot of advertising information is unable to be completed at the beginning of VIS design, but conducted gradually in some specific targeted advertising activities in the subsequent. Most traditional VIS design takes paper media as carrier, with quite a lot of restrictions to update advertising contents. Along with the application of new media, the ad production cycle is shortened obviously, making the update more easily. Therefore, the part of propaganda and advertising of VIS Design textbook for higher vocational colleges shall be compiled with emphasis.

Public relation gifts system is an effective method to promote enterprise public relations and the intermedia for the corporate to make friendly contacts to various parties. The knowledge of this part in traditional VIS Design textbook generally includes the design of notebook, bag, lighter, umbrellas, key chain, and gift bag. In information era, public relations and gifts system design need to keep in step with the times and close to life more than ever. The design of public relation gifts shall be conducted in combination with corporate culture. For example, the U disk may be taken as public relation gifts to conduct cultural and creative product design in combination with corporate mascot; as for the material, attention shall be paid to environmental protection, for example, principle of repeated use shall be reflected in the design of handbags and gift bags. Therefore, the compilation of this part in VIS Design textbook for higher vocational colleges shall strengthen the standard design of this part based on the coordination and integration of practical value and ornamental value.

Transportation system has wide scope of activities and is not limited by groups, which transmits corporate image in a mobile manner. The knowledge of this part in traditional VIS Design textbook generally includes the visual appearance design of various vehicles (car, bus, minibus, truck, tool van, bicycle etc.), planes, ships and other vehicles. In information era, taking car as an example, most vehicle-mounted systems are showing a trend of informatization. Therefore, the standard design of this part shall be compiled in VIS Design textbook for higher vocational colleges with emphasis. For instance, compile such contents as the UI interface of vehicle-mounted system, car LCD display, information auxiliary system at bus stop to the textbook.

\section{PROBLEMS NOT TO BE OVERLOOKED IN VIS DESIGN TEXTBOOK CONSTRUCTION FOR HIGHER VOCATIONAL COLLEGES IN INFORMATION ERA}

The information era has influenced the contents of VIS Design a lot, but regardless of the times progress as well as science and technology development, VIS design cannot exist in isolation from the corporate spirit and corporate culture. VIS design is not mechanical combination operation of graphic symbols, but the visual expression with concept identification as connotation. The concept identification is the soul and foundation of VIS design and the design principle, which will not change with the passage of time. VIS Design textbook construction for higher vocational colleges shall make clear the core position of concept identification for VIS design, and always conduct textbook construction with that as a guiding ideology.
New technologies constantly emerge in information era, and the application of Java language has brought dynamic performance for web pages. The promotion of Flash makes large amount of animation effects appear in web pages, and the Stream media technology has promoted the function of online play. Traditional media is far less than new media in terms of transmission speed, communication populations and interaction experience. The emergence of new technology has brought new look to the VIS design, but we shall not easily deny any kind of media pattern, nor shall we simply believe that a new one can completely replace the old one. The stability and longevity of traditional media against new media are beyond question. We shall remove the backward part of traditional VIS design with the support of existing technology, and enrich and develop the VIS design in information era using existing technology. The VIS Design textbook construction for higher vocational colleges in information era shall not break away from the basic specification of traditional VIS design system. We shall make expansion, supplement and perfection on the basis of fixed principle of traditional VIS design, forming a set of VIS design formulating standard with information characteristics, to reflect the characteristics of information era to the largest extent.

New design concepts and technical innovation has provided us with many thinking dimensions for reexamining the traditional Chinese cultural concept; the emergence of new technology and new material has provided us with more possibilities for the modern application of traditional Chinese style [5]. In information era, people pay more attention to national characteristics, local culture connotation, and regional characteristics of the industry; pay more attention to the significance of "one world, a variety of voice" for VIS design. The application of Chinese elements in modern VIS design can avoid the "integration" or "homogeneity" of culture, and can provide an unprecedented development space for Chinese VIS design. The VIS Design textbook construction for higher vocational colleges needs a new road that keeps pace with the times on the basis of national culture, to reflect the diversity of culture fully.

\section{CONCLUSION}

The arrival of information era and the progress of science and technology, constantly add new meaning and performance to VIS design. So the VIS Design textbook construction for higher vocational colleges under the background of information era shall redefine VIS design system in specific practice and application based on such background, to summarize new type of VIS design project, and clear up new contents of VIS design items; and it shall be constructed based on traditional VIS design system, to expand and enrich the existing VIS design content, and add more practical knowledge and production standard, thus to perfect the VIS Design textbooks used for Chinese higher vocational colleges, rendering it the characteristics of practice and times. 


\section{REFERENCES}

[1] Mao Feng. VI Design Basis [M].Nanjing: Jiangsu Fine Art Press, 2008.6: P003.

[2] Yu Xin. VI Design in the Context of New Media [J]. Art and Design (Theory), 2014(Z1):P40.

[3] Zhang Jun. Application and Expansion of Digital Corporate Image System in New Media Era [D].Xi'an: Xi'an Fine Arts.

[4] Zhao Xin. Application of VI Design in New Media Era [J]. New Media Research, 2015(2):53.

[5] Dou Boyan. Application of Numerology in VI System [D].Beijing: Beijing Jiaotong University, 2014:P3. 